\title{
ON THE SYMMETRY OF BIOCTONIONIC JULIA SETS
}

\author{
Andrzej Katunin \\ Institute of Fundamentals of Machinery Design, Silesian University of Technology \\ Gliwice, Poland \\ andrzej.katunin@polsl.pl
}

\begin{abstract}
The hypercomplex fractals obtained from generalizations of J- and M-sets, apart from their visual aesthetics, play an important role in the mathematical description in various fields of physics. The generalizations of J- and M-sets to the four-dimensional Euclidean space are well known and well described. However, very few studies were done for the higher-dimensional generalizations. The paper discusses the J-sets generalization to the hypercomplex algebra of bioctonions and completes the previous studies in this domain. The symmetry properties were studied for quadratic mapping of the bioctonionic J-sets. The discussion of limitations of the further generalizations of J-sets to higher hypercomplex spaces was also provided.
\end{abstract}

Keywords: Julia sets, hypercomplex fractals, bioctonions, hypercomplex algebras

\section{Introduction}

The classical Julia and Fatou sets and their generalization, Mandelbrot set, have been investigated in various domains: self-similarity, periodicity and many others. The simple recursive equation

$$
z_{k+1}=z_{k}^{2}+c
$$

where $c$ is the control parameter, which completely defines the shapes and topologies of resulting $\mathrm{J}$-sets (both parameters are complex, namely $z, c \in \mathbf{C}$ ), could generate the structures of infinitely high complexity. The generalization of the $\mathrm{J}$ - and M-sets to higher spatial dimensions is of interest to many scientists and enthusiasts. To date some generalizations are known: Pickover [1] and Norton [2] proposed the J-sets constructed using quaternions, the hypercomplex vectors presented in 4D Euclidean space, while Griffin and Joshi [3, 4] constructed J-sets using octonions, the hypercomplex vectors presented in $8 \mathrm{D}$ Euclidean space. In the latter cases the parameters of (1) are quaternions $(z, c \in \mathbf{H})$ and octonions $(z, c \in \mathbf{O})$, respectively. Further generalizations could be done using higher-dimensional Cayley-Dickson algebras, however every extension of $\mathbf{C}$ results in loss of the algebraic properties of a given algebra, which will be discussed later. 
With the increase of the dimension of a vector space, the complexity of $J$ representations increases significantly. Therefore it is necessary to investigate their symmetry properties in order to simplify the operations on them. The scientific group from the Belarussian Academy of Science studied the symmetry of J-sets based on quaternions, biquaternions [5] and octonions [6] and proved that due to the symmetry of these hypercomplex J-sets they could be defined by two numbers: the real part of the (bi)quaternionic/octonionic control parameter $C_{0}$ and the modulus of this parameter $|C|$.

Hypercomplex numbers, including J-sets, have various applications in physics theories, primarily in relativistic mechanics and kinematics. Quaternions were used in mechanics since they were introduced by Hamilton in 1843 [7]. The intensive application of the quaternions and octonions and their derivatives could be observed in electrodynamics, cosmology, quantum mechanics and special relativity [8-12]. Moreover, the investigation of hypercomplex fractal sets may be helpful in the defining of dynamic systems. The bioctonions seem to be a tool which could be used in the black holes and supergravity theories [13].

This paper deals with the special case of hypercomplex algebra - bioctonions. In the presented study the symmetry properties of J-sets constructed in such vector space were investigated based on a quadratic mapping in the form of (1). Moreover, the higher-dimensional and derivative to octonionic algebras were analyzed from the point of view of possibilities of construction of further variations of J-set.

\section{Hypercomplex algebras}

The simplest generalization of complex algebra $\mathbf{C}$ is the quaternionic algebra $\mathbf{H}$. The quaternion $z$ is a set of four real numbers (basis elements) $x_{0}, x_{1}, x_{2}, x_{3}$ with one real $e_{0}$ and three unreal units $e_{1}, e_{2}, e_{3}, e_{1}^{2}=e_{2}^{2}=e_{3}^{2}=-e_{0}=-1$ [14]. The commutation is described by the following form:

$$
z=e_{0} x_{0}+e_{1} x_{1}+e_{2} x_{2}+e_{3} x_{3}
$$

The quaternions are not commutative with respect to multiplication: $z_{1} \cdot z_{2} \neq z_{2} \cdot z_{1}$, $z_{1}, z_{2} \in \mathbf{H}$, however it holds the multiplication associativity: $\left(z_{1} \cdot z_{2}\right) \cdot z_{3}=z_{1} \cdot\left(z_{2} \cdot z_{3}\right)$. In terms of further studies it is suitable to represent (2) in the form of combination of a scalar and a vector of imaginary elements:

$$
z=x_{0}+\mathbf{x}
$$

Thus, the conjugation of a quaternion could be presented as follows:

$$
\bar{z}=\operatorname{conj}(z)=x_{0}-\mathbf{x},
$$


then

$$
z \bar{z}=\bar{z} z=|z|^{2}
$$

is a scalar and forms the modulus of $z$ :

$$
|z|=\sqrt{\sum_{i=1}^{n} x_{i}^{2}} .
$$

The next generalization of $\mathbf{C}$ is the octonionic algebra $\mathbf{O}$. The octonion $z$ could be presented in the same form as in the case of quaternions (see (2)) [14]:

$$
z=e_{0} x_{0}+e_{1} x_{1}+e_{2} x_{2}+e_{3} x_{3}+e_{4} x_{4}+e_{5} x_{5}+e_{6} x_{6}+e_{7} x_{7},
$$

where only the first element in (7) is real, thus the octonions hold the notation assumed in (3)-(6). The octonions do not hold commutativity nor associativity algebraic properties, however satisfy the weaker form of associativity - the alternativity, i.e. the subalgebra generated by two elements of $\mathbf{O}$ is associative. The algebraic properties of $\mathbf{O}$ are described by the multiplication table - Table 1 [15]. This table could also be presented by the following relations:

$$
e_{i} e_{j}=-\delta_{i j} e_{0}+\eta_{i j k} e_{k}, \quad i, j, k=1, \ldots, 7,
$$

where $\eta_{i j k}$ is a totally antisymmetric tensor for $i j k=123,145,176,246,257,347$, 365 and $e_{i} e_{0}=e_{0} e_{i}=e_{i}, e_{0} e_{0}=e_{0}$.

Table 1

Multiplication table for octonions

\begin{tabular}{|c|c|c|c|c|c|c|c|c|}
\hline$\times$ & $\boldsymbol{e}_{\mathbf{0}}$ & $\boldsymbol{e}_{\mathbf{1}}$ & $\boldsymbol{e}_{\mathbf{2}}$ & $\boldsymbol{e}_{\mathbf{3}}$ & $\boldsymbol{e}_{\mathbf{4}}$ & $\boldsymbol{e}_{\mathbf{5}}$ & $\boldsymbol{e}_{\mathbf{6}}$ & $\boldsymbol{e}_{\mathbf{7}}$ \\
\hline $\boldsymbol{e}_{\mathbf{0}}$ & $e_{0}$ & $e_{1}$ & $e_{2}$ & $e_{3}$ & $e_{4}$ & $e_{5}$ & $e_{6}$ & $e_{7}$ \\
\hline $\boldsymbol{e}_{\mathbf{1}}$ & $e_{1}$ & $-e_{0}$ & $e_{3}$ & $-e_{2}$ & $e_{5}$ & $-e_{4}$ & $-e_{7}$ & $e_{6}$ \\
\hline $\boldsymbol{e}_{\mathbf{2}}$ & $e_{2}$ & $-e_{3}$ & $-e_{0}$ & $e_{1}$ & $e_{6}$ & $e_{7}$ & $-e_{4}$ & $-e_{5}$ \\
\hline $\boldsymbol{e}_{\mathbf{3}}$ & $e_{3}$ & $e_{2}$ & $-e_{1}$ & $-e_{0}$ & $e_{7}$ & $-e_{6}$ & $e_{5}$ & $-e_{4}$ \\
\hline $\boldsymbol{e}_{\mathbf{4}}$ & $e_{4}$ & $-e_{5}$ & $-e_{6}$ & $-e_{7}$ & $-e_{0}$ & $e_{1}$ & $e_{2}$ & $e_{3}$ \\
\hline $\boldsymbol{e}_{\mathbf{5}}$ & $e_{5}$ & $e_{4}$ & $-e_{7}$ & $e_{6}$ & $-e_{1}$ & $-e_{0}$ & $-e_{3}$ & $e_{2}$ \\
\hline $\boldsymbol{e}_{\mathbf{6}}$ & $e_{6}$ & $e_{7}$ & $e_{4}$ & $-e_{5}$ & $-e_{2}$ & $e_{3}$ & $-e_{0}$ & $-e_{1}$ \\
\hline $\boldsymbol{e}_{7}$ & $e_{7}$ & $-e_{6}$ & $e_{5}$ & $e_{4}$ & $-e_{3}$ & $-e_{2}$ & $e_{1}$ & $-e_{0}$ \\
\hline
\end{tabular}

Further generalizations are possible using Cayley-Dickson construction, which could result in sedenionic (16D) complex algebra, pathionic (32D) complex algebra, chingonic (64D), etc., however the algebras beyond octonions do not satisfy even alternativity algebraic property and thus cannot be composition algebras. 
Another algebras could be constructed by using tensor product of the abovementioned ones [15]. In the following way the biquaternions $\mathbf{C} \otimes \mathbf{H}$ could be considered, which consider the complexes of $\mathbf{C}$ and reals of $\mathbf{H}$. This algebra holds the properties of $\mathbf{H}$, i.e. it is non-commutative and associative. Moreover it fulfills the multiplication properties of octonions, i.e. the multiplication table (see Table 1) is the same for $\mathbf{C} \otimes \mathbf{H}$. The same operation forms the bioctonionic algebra $\mathbf{C} \otimes \mathbf{O}$, which holds properties of $\mathbf{O}$. Other combinations are possible, e.g. quateroctonions $\mathbf{H} \otimes \mathbf{O}$ or octooctonions $\mathbf{O} \otimes \mathbf{O}$, however such algebras are not alternative, i.e. there exist two elements of each of these algebras, which does not continue the associative subalgebra [16].

\section{Bioctonionic J-set and other generalizations}

The biquaternions introduced in [1] have the modified structure of (3):

$$
z=\varepsilon x_{0}+\mathbf{x}
$$

where parameter $\varepsilon$ could assume three values: $\varepsilon^{2}=-1$ (ordinary complex numbers), $\varepsilon^{2}=1$ (double numbers) and $\varepsilon^{2}=0$ (dual numbers) [17]. Such an approach could be generalized to bioctonions. The equation (1) for them takes the form:

$$
z_{k+1} \rightarrow \varepsilon z_{k}^{2}+c, z, c \in \mathbf{C} \otimes \mathbf{O}
$$

The equation (10) maps 8D Euclidean space $\left(\varepsilon^{2}=1\right)$, 8D Galilei-Newton space $\left(\varepsilon^{2}=0\right)$ and 8D Minkowski space $\left(\varepsilon^{2}=-1\right)$ on itself $(\mathrm{cf} .[5,17])$. The following cases generated by (10) could be presented:

$$
\begin{gathered}
-z_{k+1} \rightarrow-\varepsilon z_{k}^{2}-c, \\
\bar{z}_{k+1} \rightarrow \varepsilon \bar{z}_{k}^{2}+\bar{c}, \\
-\bar{z}_{k+1} \rightarrow-\varepsilon \bar{z}_{k}^{2}-\bar{c},
\end{gathered}
$$

where $\bar{c}=c_{0}+\mathbf{c}$. The sets (11)-(13) are invariant under a reflection defined by the bioctonion conjugation. Moreover, there is additional invariance:

$$
z_{k}^{\prime}=o z_{k} \bar{O}, \varepsilon_{k}^{\prime}=o \varepsilon_{k} \bar{O}, c_{k}^{\prime}=o c_{k} \bar{O}, o \bar{O}=1
$$

This implies the equivalent form of (10):

$$
z_{k+1}^{\prime} \rightarrow \varepsilon^{\prime}\left(z_{k}^{\prime}\right)^{2}+c^{\prime} .
$$


If all points $z_{0} \in \mathbf{C}$, then the points $z_{k} \in \mathbf{C}$ for the Julia set $J_{\mathbf{C}}$, thus $J_{\mathbf{C}} \subseteq J_{\mathbf{C} \otimes \mathbf{o}}$. Considering (14), which defines the symmetries of $J_{\mathbf{C} \otimes \mathbf{O}}$, the bioctonion $O$ takes form (see [18]):

$$
o=\frac{1+\frac{\mathbf{c}}{|\mathbf{c}|} \tan \frac{\varphi}{2}}{\sqrt{1+\tan ^{2} \frac{\varphi}{2}}}=\cos \frac{\varphi}{2}+\frac{\mathbf{c}}{|\mathbf{c}|} \sin \frac{\varphi}{2},
$$

where $\varphi$ is the angle of rotation. Equation (16) shows, that the above-presented constructions are rotation-invariant. Hence, the $J_{\mathbf{C} \otimes \mathbf{O}}$ could be fully defined by two numbers, $c_{0}$ and $|\mathbf{c}|$.

\section{Conclusions}

The paper presented the analysis of existence and symmetry properties of bioctonionic J-sets. The bioctonionic algebras could be used for construction of three types of equations for the generation of $8 \mathrm{D}$ hypercomplex J-sets and it was shown that the further generalization of $\mathrm{J}$-sets on higher-dimensional hypercomplex algebras and alternative algebras, like quateroctonions and octooctonions, is not possible because of loss of their algebraic properties.

\section{References}

[1] Pickover C.A., Visualization of quaternion slices, Image Vision Comput. 1988, 6, 235-237.

[2] Norton A., Julia sets in the quaternions, Comput. Graph. 1989, 2, 267-278.

[3] Griffin C.J., Joshi G.C., Octonionic Julia sets, Chaos Soliton. Fract. 1992, 11-24.

[4] Griffin C.J., Joshi G.C., Transition points in octonionic Julia sets, Chaos Soliton. Fract. 1993, 67-88.

[5] Bogush A.A., Gazizov A.Z., Kurochkin Yu.A., Stosui V.T., Symmetry properties of quaternionic and biquaternionic analogs of Julia sets, Ukr. J. Phys. 2003, 48, 295-299.

[6] Kurochkin Yu.A., Zhukovich S.Ya., Set symmetry, generated by octonion analog of Julia-Fatou algorithm, Vestn. Brest. U. 4 - Phys. Math. 2010, 2, 74-79 (in Russian).

[7] Mukundan R., Quaternions: from classical mechanics to computer graphics, and beyond, Proc. $7^{\text {th }}$ Asian Technology Conference in Mathematics, Melaka 2002, 97-106.

[8] Dang Y., Kauffman L.H., Sandin D.J., Hypercomplex Iterations: Distance Estimation and Higher Dimensional Fractals, World Scientific, Chicago 2002.

[9] Yefremov A.P., Bi-quaternion square roots, rotational relativity, and dual space-time intervals, AIP Conf. Proc. 2007, 13, 178-184.

[10] Yefremov A.P., Fundamental properties of quaternion spinors, AIP Conf. Proc. 2012, 18, 188-195. 
[11] Okubo S., Introduction to Octonion and Other Non-Associative Algebras in Physics, Cambridge University Press, Cambridge 1995.

[12] Baez J.C., The octonions, Bull. Amer. Math. Soc. 2002, 39, 145-205.

[13] Rios M., Jordan C*-algebras and supergravity, arXiv:1005.3514, 2010.

[14] Conway J.H., Smith D.A., On Quaternions and Octonions: Their Geometry, Arithmetic and Symmetry, Peters, 2003.

[15] Sabadini I., Shapiro M.V., Sommen F., Hypercomplex Analysis, Springer, Berlin 2009.

[16] Rosenfeld B.A., Geometry of Lie groups, Springer, Dordrecht 1997.

[17] Yaglom I.M., Complex Numbers and Its Application in Geometry, Fizmatgiz, Moscow 1963 (in Russian).

[18] Berezin A.V., Kurochkin Yu.A., Tolkachev E.A., Quaternions in Relativistic Physics, Nauka i Tekhnika, Minsk 1989 (in Russian). 